\title{
Effect of Thickness and Perforation Size on the Acoustic Absorption Performance of a Micro- Perforated Panel
}

\author{
Yi-San Wong ${ }^{1}$, Vignesh Sekar ${ }^{1}$, Se Yong Eh Noum ${ }^{1, *}$, and Sivakumar Sivanesan ${ }^{1}$ \\ ${ }^{1}$ Taylor's University, School of Computer Science and Engineering, No. 1, Jalan Taylor's, 47500 \\ Selangor, Malaysia
}

\begin{abstract}
In current times, noise pollution is especially apparent in urban areas due to rapid development in transportation, industrialization, and urbanization. The worsening noise pollution is detrimental to human health and behaviour as it can contribute to disorders and psychological disturbance. Thus, noise regulation is crucial and must be addressed with immediate effect. Micro-perforated panels (MPP) can be a potential solution to mitigate noise on a commercial scale. Researchers have addressed the mechanics behind the enhancement of acoustic absorption through microperforation and some suggestions have been made, such as the effect of structural variation on sound absorption performance. Hence, this research aims at optimizing the sound absorption performance of an MPP by determining the connection between thickness and perforation size with sound absorption coefficient. Three cases were considered: (i) varying perforation size, (ii) varying thickness, and (iii) varying perforation size and thickness simultaneously. Based on the Maa prediction model, the sound absorption performance for all three cases have been simulated through the MATLAB software. Results show that the increase in both thickness and perforation size together increases the peak value of sound absorption coefficient (SAC). It also shifts the peak towards the higher frequency region and narrows the bandwidth. The findings of this study indicate the potential of thick MPPs as commercial sound absorbers by adjusting the size of perforations. Thicker and sturdier MPPs with optimal acoustic resistance and reactance can act as reliable sound absorbers for sound insulation purposes.
\end{abstract}

\section{Introduction}

In recent times, noise pollution is one of the biggest issues faced by humankind as it affects both mental and physical health and poses psychological risks [1]. Sound generated from dynamic sources, such as traffic and crowds, is a major contribution to noise pollution in urban areas. Generally, noise is an undesirable sound that is loud, unpleasant and causes disturbance. Noise is conventionally quantified by frequency in $\operatorname{Hertz}(\mathrm{Hz})$ and sound pressure level in decibels $(\mathrm{dB}$ and $\mathrm{dBA})$. According to the Malaysian Department of

\footnotetext{
* Corresponding author: SeYong.EhNoum@taylors.edu.my
} 
Environment's Guidelines for Environmental Noise Limits and Control, the maximum permissible sound levels for low, medium, and high-density residential areas range from 55 dBA to $65 \mathrm{dBA}$ [2]. From a 2018 study conducted at two road intersections leading to nearby residential units in Shah Alam, the highest recorded noise level in a day was well above 90 dBA which greatly exceeded the allowable limit [3]. On top of that, Malaysia has an annual growth rate of approximately 6 percent in terms of the number of registered private vehicles [4]. The constant or prolonged exposure to noise may lead to hearing impairment on top of triggering numerous stress responses and altering normal circadian rhythms [5]. Hence, noise regulation is crucial and is preferably resolved using natural and waste material to minimise environmental impact. Commercially available sound absorbing materials are usually porous, and these materials can be categorised as granular, cellular, or fibrous [6]. Fibrous materials consist of many openings, formed by gaps in between fibres, which absorb and dissipate sound waves. Internal friction in the fibrous material causes the reduction of sound energy through thermal and viscous losses [7]. Fibrous materials can be further classified as either natural or synthetic. Currently, the latter is commonly used as a commercial sound absorbing material such as glass-fibre and mineral fibre [8]. Although synthetic fibres are superior in terms of acoustic performance due to their smaller diameter and antifungal quality, they affect the environment significantly more than natural fibres [9]. The biodegradable and lightweight nature as well as the affordability of natural fibres have made them a more favourable option in acoustic absorption purposes, especially for low frequency regions or residential settings [1]. As an alternative to regular acoustic panels, micro-perforated panels (MPP), as depicted in Figure 1, have been developed and applied for the purpose of noise dampening as well. Simply put, MPP is a panel (typically a thin one) with sub-millimetre perforations backed by an air gap and a rigid wall [10]. MPPs are commonly made from metallic materials such as aluminium and steel in which their production processes release a significant amount of carbon to the atmosphere. Moreover, the fabrication of MPP from metallic material is costly due to the complex process of producing sub-millimetre perforations using etching, jetting, or laser technology [11]. By adjusting design parameters such as panel thickness and perforation size, the optimal sound absorption performance of an MPP can be achieved with fewer negative effects to the environment, economy, and society [12].

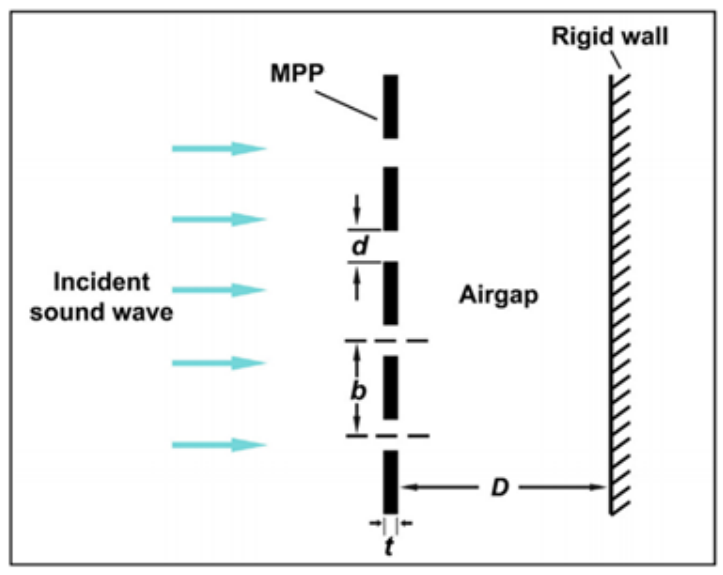

Fig. 1. Setup of an MPP

It can be deduced that sound absorption performance is largely related to the microscopic layout in the acoustic panel itself. In most cases, key parameters are interdependent which renders it difficult to determine the optimal conditions through experimental method. As shown in Table 1, researchers have discussed the effect of adjusting 
a single parameter on the sound absorption performance of an acoustic panel. However, they have yet to explore the effect of simultaneously altering multiple parameters, specifically panel thickness and perforation size, on the sound absorption performance of an MPP. Hence, the use of $3 \mathrm{D}$ or computer simulation for better visualisation will be greatly appreciated. This research aims at determining the correlation between panel thickness and perforation size in optimizing the sound absorption performance of an MPP through MATLAB simulation. Thus, the findings of this study can contribute to the improvement in the design of MPPs for effective sound absorption.

Table 1. Research gap for studies on MPP

\begin{tabular}{|c|c|c|c|}
\hline Parameter & Source(s) & Key Findings & Research Gap \\
\hline Thickness & [14] [15] & $\begin{array}{l}\text { Thicker panels present a lower } \\
\text { peak of sound absorption } \\
\text { coefficient. }\end{array}$ & \multirow[b]{2}{*}{$\begin{array}{l}\text { The effect of a concurrent } \\
\text { increase in thickness and } \\
\text { perforation size on the peak } \\
\text { of sound absorption } \\
\text { coefficient. }\end{array}$} \\
\hline $\begin{array}{l}\text { Perforation } \\
\text { size }\end{array}$ & [16] & $\begin{array}{l}\text { Larger } \\
\text { perforation sizes present a } \\
\text { higher peak of sound } \\
\text { absorption coefficient up until } \\
\text { an extent where the peak value } \\
\text { declines. }\end{array}$ & \\
\hline
\end{tabular}

\subsection{Theory of MPP}

An MPP consists of small perforations whereby each of them acts as a single Helmholtz resonator and dissipates sound energy mostly through resonance and viscous loss in the neck of the perforation. Similar to a mass-spring system, the perforations of an MPP represent the acoustic mass while the air gap embodies the acoustic spring. The incident sound waves from a certain source drive the air molecules in the perforations to oscillate back and forth, which results in an energy loss or acoustic absorption. This can be observed when the reflected sound energy is lower than the incident sound energy. The absorption comes from an MPP's acoustic impedance which consists of resistance (real) and reactance (imaginary) components [17]. In the case where the incident sound wavelength is greater than the perforation diameter and perforation distance, the acoustic impedance of a perforation can be defined as [18]:

$$
\begin{aligned}
& Z=j \omega \rho t\left[1-\left(\frac{2}{\xi \sqrt{-j}}\right)\left(\frac{J_{1}(\xi \sqrt{-j})}{J_{0}(\xi \sqrt{-j})}\right)\right]^{-1} \\
& \xi=r \sqrt{\frac{\rho \omega}{\eta}}
\end{aligned}
$$

where $\mathrm{Z}$ is the acoustic impedance of perforation, $\omega$ is the angular frequency of the sound, $\rho$ is the density of air, $t$ is the panel thickness, $r$ is the perforation radius, $\eta$ is the air dynamic viscosity, and J_0 and J_1 are the zeroth and first order for Bessel functions. To obtain the acoustic impedance of the MPP system, Equation (1) is divided by the perforation ratio, which is the ratio of perforated area to the surface area of the MPP.

However, the acoustic impedance formula in Equation (1) is impractical to use. Maa suggested a better alternative that is effective for lower perforation ratios and only has around $5 \%$ error [20]. Hence, acoustic impedance can be better expressed as:

$$
\begin{aligned}
& Z_{N}=R+j M \\
& R=\frac{C_{1} t \times 10^{-5}}{p d^{2}}\left(\sqrt{1+\frac{x^{2}}{32}}+\frac{x d \sqrt{2}}{8 t}\right)
\end{aligned}
$$




$$
\begin{aligned}
& M=0.0185 \frac{t f}{p}\left(1+\frac{1}{\sqrt{9+\frac{x^{2}}{2}}}+\frac{0.85 d}{t}\right) \\
& x=C_{2} \times 10^{-3} d \sqrt{f} \\
& p=\frac{\pi}{4}\left(\frac{d}{b}\right)^{2}
\end{aligned}
$$

where $Z_{N}$ is the normalised acoustic impedance, $\mathrm{R}$ and $\mathrm{M}$ are the resistance and reactance components, $C_{1}$ and $C_{2}$ are constants of 0.147 and 0.316 for a non-metallic MPP, $\mathrm{f}$ is the sound frequency, $\mathrm{d}$ is the perforation diameter, $\mathrm{p}$ is the perforation ratio, and $\mathrm{b}$ is the perforation distance. The sound absorption coefficient (SAC) of an MPP backed by an air gap can then be obtained by [19]:

$$
\alpha=\frac{4 R}{(1+R)^{2}+\left[\omega M-\cot \left(\frac{\omega D}{c_{0}}\right)\right]^{2}}
$$

where $\alpha$ is the SAC, D is the air gap distance, and $c_{0}$ is the speed of sound.

\section{Method}

\subsection{MATLAB analysis}

A MATLAB simulation was performed to determine the effect of thickness and perforation size on the sound absorption performance of an MPP. Equation (8) was translated into MATLAB and the SAC of the MPPs were computed. The frequency range was set from 500 $\mathrm{Hz}$ to $4500 \mathrm{~Hz}$ to mimic the standard frequency range of a speaker used in an impedance tube test [20] [21]. The speed of sound used was $343 \mathrm{~m} / \mathrm{s}$ and the air gap distance was fixed at 1 $\mathrm{mm}$. Three cases were considered: (i) varying perforation size with constant thickness, (ii) varying thickness with constant perforation size, and (iii) varying perforation size and thickness simultaneously. The parameters for Case 1, Case 2, and Case 3 are presented in Table 2, Table 3, and Table 4, respectively. The SACs of the MPPs were presented in graphs

\begin{tabular}{|c|c|c|c|}
\hline Thickness (mm) & $\begin{array}{c}\text { Perforation Size } \\
(\mathbf{m m})\end{array}$ & $\begin{array}{c}\text { Perforation Distance } \\
(\mathbf{m m})\end{array}$ & $\begin{array}{c}\text { Perforation Ratio } \\
(\%)\end{array}$ \\
\hline \multirow{4}{*}{2} & 0.2 & \multirow[t]{4}{*}{ 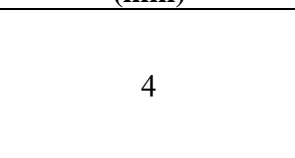 } & 0.2 \\
\hline & 0.3 & & 0.5 \\
\hline & 0.4 & & 0.8 \\
\hline & 0.5 & & 1.3 \\
\hline \multirow{4}{*}{3} & 0.2 & \multirow{4}{*}{4} & 0.2 \\
\hline & 0.3 & & 0.5 \\
\hline & 0.4 & & 0.8 \\
\hline & 0.5 & & 1.3 \\
\hline \multirow{3}{*}{4} & 0.2 & \multirow{3}{*}{4} & 0.2 \\
\hline & 0.3 & & 0.5 \\
\hline & 0.4 & & 0.8 \\
\hline
\end{tabular}
and thoroughly discussed to evaluate the significance of the change in thickness and perforation size on the sound absorption performance of an MPP.

Table 2. Case 1 (varying perforation size with constant thickness) 


\begin{tabular}{|c|c|c|c|}
\hline & 0.5 & & 1.3 \\
\hline \multirow{4}{*}{5} & 0.2 & \multirow{4}{*}{4} & 0.2 \\
\hline & 0.3 & & 0.5 \\
\hline & 0.4 & & 0.8 \\
\hline & 0.5 & & 1.3 \\
\hline
\end{tabular}

Table 3. Case 2 (varying thickness with constant perforation size)

\begin{tabular}{|c|c|c|c|}
\hline $\begin{array}{c}\text { Perforation Size } \\
(\mathbf{m m})\end{array}$ & Thickness (mm) & $\begin{array}{l}\text { Perforation Distance } \\
(\mathbf{m m})\end{array}$ & $\begin{array}{c}\text { Perforation Ratio } \\
(\%)\end{array}$ \\
\hline \multirow[t]{4}{*}{ (2min) } & 2 & \multirow[t]{4}{*}{ 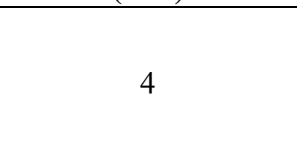 } & \multirow[t]{4}{*}{$x_{2}$} \\
\hline & 3 & & \\
\hline & 4 & & \\
\hline & 5 & & \\
\hline \multirow{4}{*}{0.3} & 2 & \multirow{4}{*}{4} & \multirow{4}{*}{0.5} \\
\hline & 3 & & \\
\hline & 4 & & \\
\hline & 5 & & \\
\hline \multirow{4}{*}{0.4} & 2 & \multirow{4}{*}{4} & \multirow{4}{*}{0.8} \\
\hline & 3 & & \\
\hline & 4 & & \\
\hline & 5 & & \\
\hline \multirow{4}{*}{0.5} & 2 & \multirow{4}{*}{4} & \multirow{4}{*}{1.3} \\
\hline & 3 & & \\
\hline & 4 & & \\
\hline & 5 & & \\
\hline
\end{tabular}

Table4. Case 3 (varying perforation size and constant thickness simultaneously)

\begin{tabular}{|c|c|c|c|}
\hline Thickness $(\mathbf{m m})$ & $\begin{array}{c}\text { Perforation Size } \\
(\mathbf{m m})\end{array}$ & $\begin{array}{c}\text { Perforation Distance } \\
(\mathbf{m m})\end{array}$ & $\begin{array}{c}\text { Perforation Ratio } \\
(\boldsymbol{\%})\end{array}$ \\
\hline 2 & 0.2 & & 0.2 \\
\hline 3 & 0.3 & \multirow{3}{*}{4} & 0.5 \\
\hline 4 & 0.4 & & 0.8 \\
\hline 5 & 0.5 & & 1.3 \\
\hline
\end{tabular}

\section{Results and Discussion}

\subsection{Effect of perforation size with constant thickness on acoustic absorption}

Based on the Maa model, the computed SACs for Case 1 at a frequency range of $500 \mathrm{~Hz}$ to $4500 \mathrm{~Hz}$ are as shown in Figure 2. Results show that for a constant MPP thickness, the increase in perforation size leads to an increase in peak SAC. For all values of thickness from $2 \mathrm{~mm}$ to $5 \mathrm{~mm}$, the highest SAC is attained by the largest perforation size which is $0.5 \mathrm{~mm}$. The highest SAC recorded overall is approximately 0.86 achieved by the $2 \mathrm{~mm}$ thick MPP with $0.5 \mathrm{~mm}$ perforation size within the frequency range of $3500 \mathrm{~Hz}$ to $4000 \mathrm{~Hz}$. From Figure 2(b), the highest SAC for $3 \mathrm{~mm}$ thickness is approximately 0.76 within the frequency range of $3000 \mathrm{~Hz}$ to $3500 \mathrm{~Hz}$. Figure 2(c) shows that a $4 \mathrm{~mm}$ thick MPP can achieve a peak SAC of approximately 0.69 at a frequency between $2500 \mathrm{~Hz}$ to $3000 \mathrm{~Hz}$. As for $5 \mathrm{~mm}$ thickness, the highest SAC obtained is approximately 0.62 at a frequency of around $2500 \mathrm{~Hz}$.

Another observation is that the increase in perforation size shifts the peak SAC toward the higher frequency region and narrows the bandwidth for effective sound absorption. 
Hence, it can be deduced that a constant MPP thickness with a larger perforation size produces a high SAC peak in the higher frequency region and a narrower bandwidth. Unlike larger perforations, smaller perforations lack the acoustic resistance to absorb sound and their size also makes it more difficult for sound waves to enter. This results in most of the incident sound waves being reflected on the MPP surface instead of being absorbed. Larger perforations provide better absorption in the high frequency region as they increase the perforation ratio and reduce the total acoustic mass, which in turn increases the resonant frequency where the peak SAC occurs $[22,23]$.

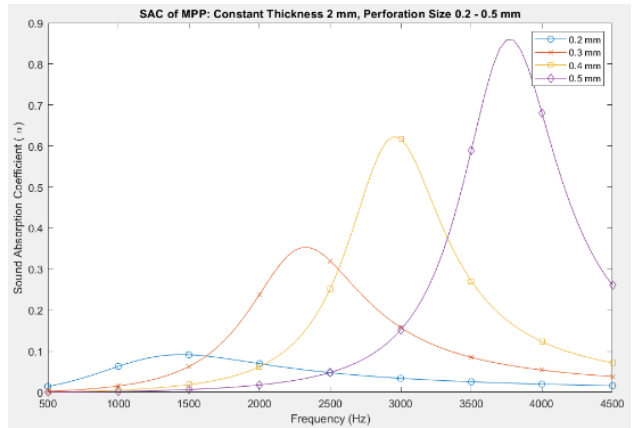

(a)

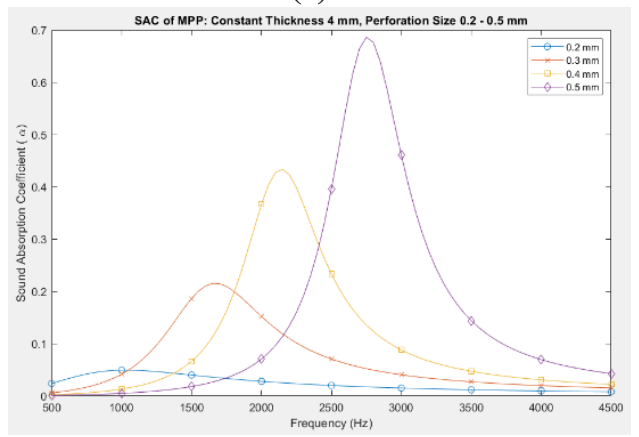

(c)

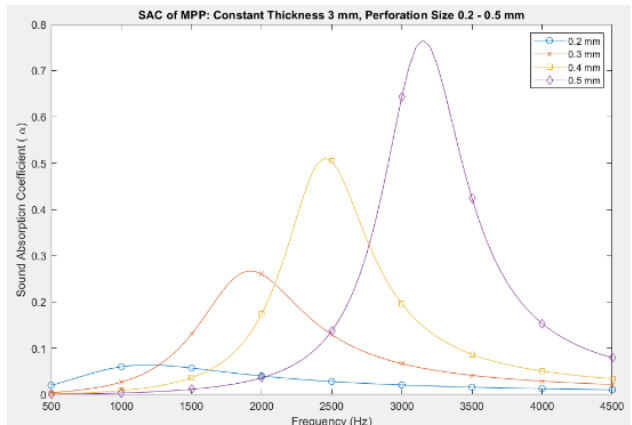

(b)

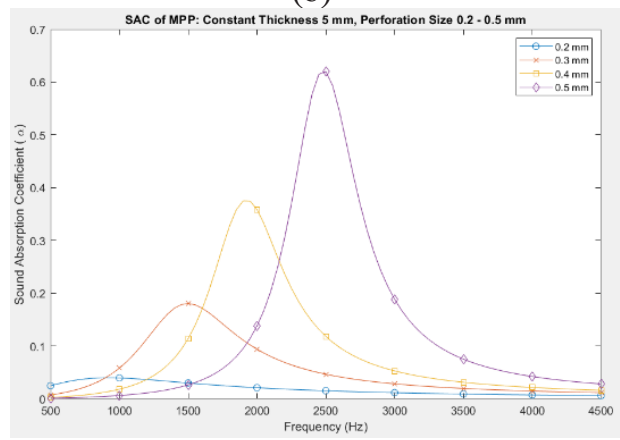

(d)

Fig. 2. SAC for Case 1: varying perforation size with constant thickness of (a) $2 \mathrm{~mm}$, (b) $3 \mathrm{~mm}$, (c) 4 $\mathrm{mm}$, and (d) $5 \mathrm{~mm}$

The findings are supported by results from a past study which indicate that the increase in perforation diameter improved the SAC value of an MPP and shifted the sound absorption peak towards the high frequency region [16]. However, it is important to note that the peak SAC value reduces as the perforation diameter further increases. An excessively large perforation size causes a reduction in acoustic resistance which reduces sound absorption performance. In contrast, minimizing the perforation size creates difficulty for sound waves to enter, hence sound absorption becomes less effective since most of the sound waves are reflected on the surface of the MPP.

\subsection{Effect of thickness with constant perforation size on acoustic absorption}

Based on the Maa model, the computed SACs for Case 2 at a frequency range of $500 \mathrm{~Hz}$ to $4500 \mathrm{~Hz}$ are as shown in Figure 3. The graphs show that for a constant perforation size, the increase in MPP thickness results in the decrease of peak SAC. For all values of perforation size from $0.2 \mathrm{~mm}$ to $0.5 \mathrm{~mm}$, the highest SAC is attained by the thinnest MPP which is 2 $\mathrm{mm}$. Similar to Case 1 , the highest SAC recorded overall is approximately 0.86 achieved by 
the $2 \mathrm{~mm}$ thick MPP with $0.5 \mathrm{~mm}$ perforation size within the frequency range of $3500 \mathrm{~Hz}$ to $4000 \mathrm{~Hz}$. From Figure 3(a), the highest SAC for $0.2 \mathrm{~mm}$ perforation size is approximately 0.09 within the frequency range of $1000 \mathrm{~Hz}$ to $2000 \mathrm{~Hz}$. Figure 3(b) shows that an MPP with $0.3 \mathrm{~mm}$ perforation size can achieve a peak SAC of approximately 0.35 at a frequency between $2000 \mathrm{~Hz}$ to $3000 \mathrm{~Hz}$. As for $0.4 \mathrm{~mm}$ perforation size, the highest SAC obtained is approximately 0.63 at a frequency in between $2500 \mathrm{~Hz}$ to $3500 \mathrm{~Hz}$.

Another observation is that the increase in MPP thickness shifts the peak SAC toward the lower frequency region but does not considerably affect the width of frequency spectrum for effective sound absorption. Hence, it can be inferred that a thinner MPP with constant perforation size reaches a high SAC peak at a higher frequency without significantly affecting the bandwidth for effective sound absorption. Unlike a thinner MPP, a thicker MPP creates a longer throat or perforation neck which provides excessive acoustic resistance and reduces the peak SAC value. A thinner MPP provides better absorption in the high frequency region as it reduces the total acoustic mass, leading to an increase in the resultant resonant frequency where the peak SAC occurs. In general, thicker panels perform better for low frequency sounds while thinner panels perform better for high frequency sounds.

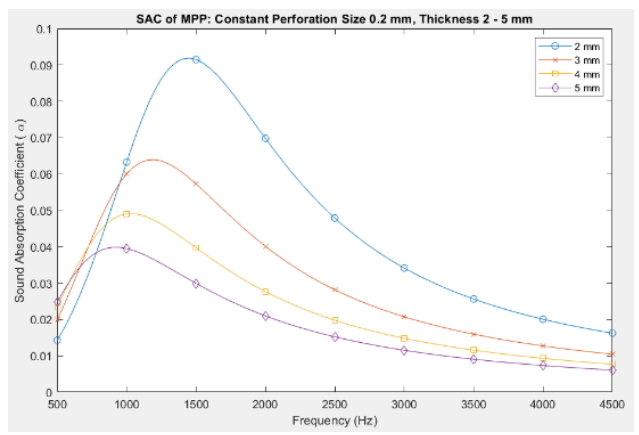

(a)

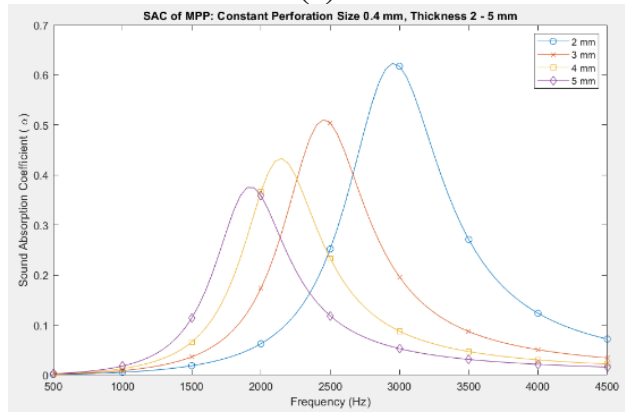

(c)

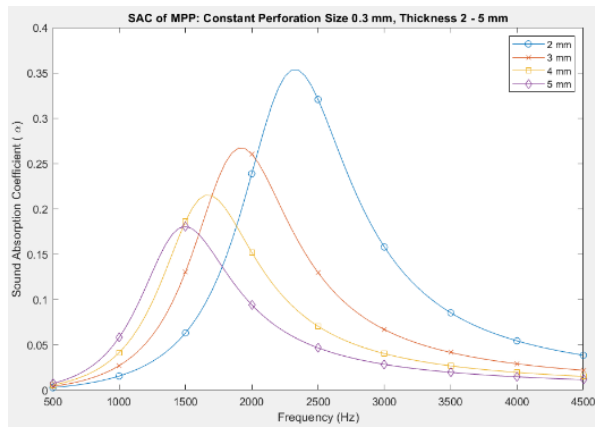

(b)

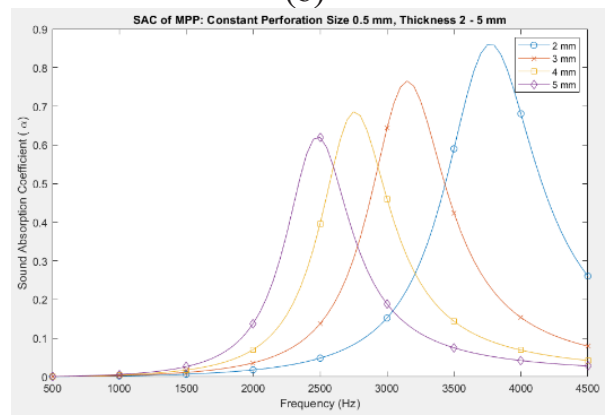

(d)

Fig. 3. SAC for Case 2: varying thickness with constant perforation size of (a) $0.2 \mathrm{~mm}$, (b) $0.3 \mathrm{~mm}$, (c) $0.4 \mathrm{~mm}$, and (d) $0.5 \mathrm{~mm}$

These findings agree with results from a past study which deduced that a thick panel with small perforations tends to have low acoustical performance due to an exceedingly large acoustic resistance in the neck of the perforations [15]. It was reported that the increase in thickness without altering the hole diameter reduces the SAC and shifts the peak to a lower frequency. This is because the total mass of air in the perforations increases as the MPP becomes thicker, thus producing a lower resonant frequency. Another research has also established that thin panels tend to absorb sounds with a shorter wavelength (high frequency), while thick panels are inclined to absorb sounds with a longer wavelength (low frequency) [24]. 


\subsection{Relationship between perforation size and thickness with acoustic absorption}

Based on the Maa model, the computed SACs for Case 3 at a frequency range of $500 \mathrm{~Hz}$ to $4500 \mathrm{~Hz}$ are as shown in Figure 4. Results show that increasing both the MPP thickness and perforation size leads to an increase in peak SAC. Overall, the highest SAC recorded is approximately 0.63 achieved by the $5 \mathrm{~mm}$ thick MPP with $0.5 \mathrm{~mm}$ perforation size at a frequency of around $2500 \mathrm{~Hz}$. The peak SAC for $2 \mathrm{~mm}$ thickness with $0.2 \mathrm{~mm}$ perforation size is approximately 0.09 within the frequency range of $1000 \mathrm{~Hz}$ to $2000 \mathrm{~Hz}$. On the other hand, a $3 \mathrm{~mm}$ thick MPP with $0.3 \mathrm{~mm}$ perforation size can achieve a peak SAC of approximately 0.27 at a frequency of around $2000 \mathrm{~Hz}$. As for $4 \mathrm{~mm}$ thickness with $0.4 \mathrm{~mm}$ perforation size, the highest SAC obtained is approximately 0.44 within the frequency range of $2000 \mathrm{~Hz}$ to $2500 \mathrm{~Hz}$.

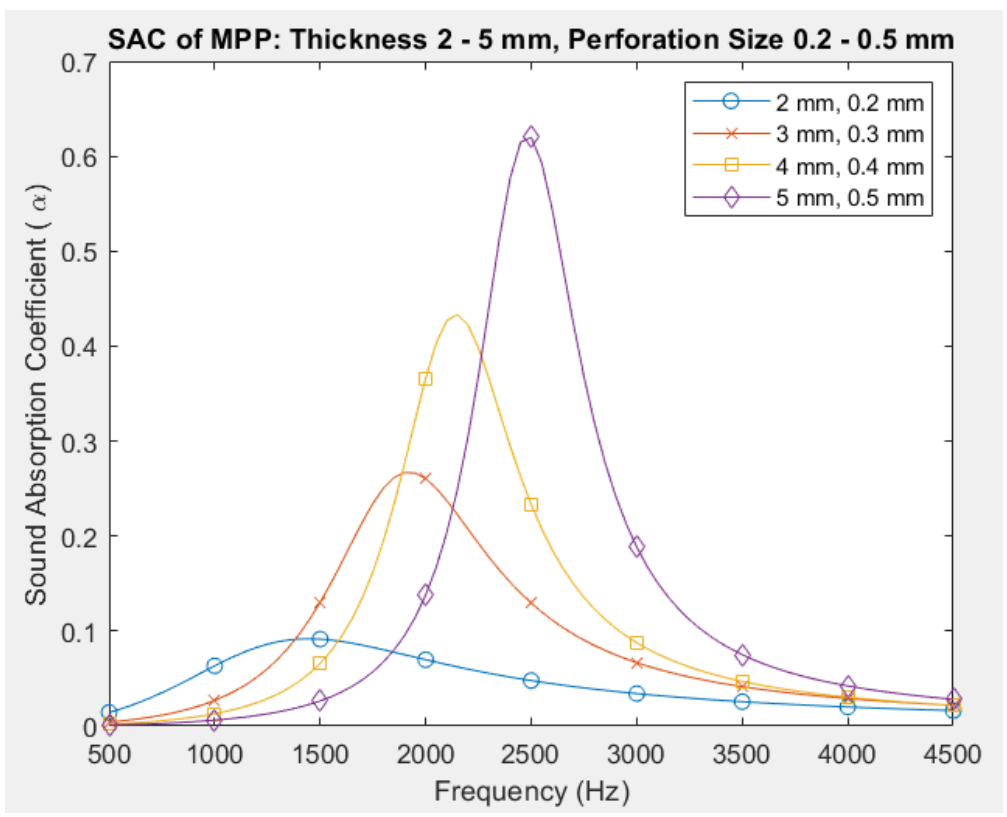

Fig. 4. Comparison of all SACs in Case 3

From Figure 4, it can be observed that the increase in both parameters increases the peak SAC, shifts it toward the higher frequency region and narrows the bandwidth. This is because increasing the thickness and perforation size provides optimistic acoustic resistance and reactance, which in turn increases the acoustic absorption. MPPs are usually thin and unreliable in terms of mechanical properties and strength, hence researchers had initially suggested increasing the thickness of an MPP for a given perforation size. However, they found that this gave an unsatisfactory acoustic absorption performance. A past paper pointed out that the absorption performance of a conventional MPP with a thickness of $0.4 \mathrm{~mm}$ significantly dropped when its thickness was increased to $10 \mathrm{~mm}$, given that the perforation diameter remained unchanged [15]. Hence, results from this study demonstrate the potential of a thicker MPP as an effective sound absorber by increasing its perforation size simultaneously. These MPPs with optimised parameters can produce optimal acoustic resistance and reactance, making them reliable both in terms of their acoustic and mechanical properties. 


\section{Conclusion}

Based on the study, it is evident that the peak SAC is significantly influenced by the alteration of perforation size and thickness of an MPP. Moreover, these parameters also affect the frequency region of the peak SAC and the effective bandwidth. Although the increase in perforation size leads to an increase in peak SAC, it is the opposite case for increasing thickness. The former also moves the peak SAC to higher frequencies, while the latter shifts the peak SAC to lower frequencies. In terms of frequency bandwidth, it becomes narrower with the increase in perforation size but is inconsiderably affected by panel thickness. Results from Case 3 demonstrated that increasing both parameters result in the increase in peak SAC towards a higher frequency with a narrower bandwidth. The MPP with $5 \mathrm{~mm}$ thickness and $0.5 \mathrm{~mm}$ perforation size achieved the best peak absorption of 0.63 at an approximate frequency of $2500 \mathrm{~Hz}$. The findings of this study indicate the potential of thick MPPs as commercial sound absorbers by adjusting the perforation size. Thicker and sturdier MPPs with optimal acoustic resistance and reactance can act as reliable sound absorbers for sound insulation in buildings. Future work involves developing a thicker MPP with an appropriate perforation size and testing its acoustic absorption performance using a two-microphone impedance tube method and/or a 3D or Finite Element Analysis (FEA) simulation.

The authors would like to thank Taylor's University and the School of Engineering staff for providing the necessary facilities and resources for this research.

\section{References}

1. N. Bhingare, S. Prakash, V. Jatti, Polym. Test., 80 (2019)

2. Anonymous, Guidelines for Environmental Noise Limits and Control, (Department of Environment Malaysia, Putrajaya, 2019)

3. I. Izzaty Mohd Isa, Z. Zafflina Mohd Zaki, J. Kassim, Int. J. Eng. \& Tech., 7, 311 (2018)

4. M. Solah, A. Hamzah, A. Ariffin, N. Paiman, I. Abdul Hamid, M. Abdul Wahab, Z. Mohd Jawi, M. Osman, J. Soc. Auto. Eng. Mas., 1, 3 (2017)

5. C. Echeverria, F. Pahlevani, W. Handoko, C. Jiang, C. Doolan, V. Sahajwalla, Resour., Cons. \& Recy., 143 (2019)

6. J. Arenas, M. Crocker, Sou. \& Vibr., 44, 7 (2010)

7. W. Yang, Y. Li, Sci. Ch. Tech. Sci., 55, 8 (2012)

8. E. Samsudin, L. Ismail, A. Abdul Kadir, J. Eng. \& App. Sci., 11, 6 (2016)

9. M. Mohd Azahari, A. M. Rus, S. Kormin, M. Zaliran, Malay. J. Ana. Sci, 22, 6 (2018)

10. D. Herrin, W. Liu, X. Hua, J. Liu, Sou. \& Vibr., 51, 12 (2017)

11. D. Chin, M. Yahya, N. Che Din, P. Ong, App. Acou., 138 (2018)

12. Y. Wang, S. Liu, H. Wu, C. Zhang, J. Xu\& H. Yu, e-Poly., 20, 1 (2020)

13. Z. Liu, J. Zhan, M. Fard, J. Davy, App. Acou., 121 (2017)

14. I. Prasetiyo, J. Sarwono, I. Sihar, J. Mech. Eng. \& Sci., 10, 3 (2016)

15. K. Sakagami, M. Morimoto, M. Yairi \& A. Minemura, App. Acou., 69, 2 (2008)

16. N. Hamdan, M. Zainulabidin, M. Kasron, A. Ismail \& A. Kassim, Int. J. Integ. Eng., 10, 4 (2018)

17. N. Jafar, L. Ooi, A. Mazlan, K. Seng J. Tan, IOP Conf. Ser.: Mat Sci. \& Eng., 815 (2020)

18. I. Crandall, Theory of vibrating systems and sound (D. Van Nostrand Company Inc., New York, 1927) 
19. D. Maa, Scientia Sinica, 18, 1 (1975)

20. S. Shuib, M. Yahya, A. Abdullah, N. Kamaruddin, J. Mech. Eng., 5, 5 (2018)

21. K. Or, A. Putra, M. Selamat, App. Acou., 119 (2017)

22. W. Tan, R. Haslina, E. Lim., H. Chuah, IOP Conf. Ser.: Mat. Sci. \& Eng., 670 (2019)

23. G. Vitkauskaite, R. Grubliauskas, Ener. Pro., 147 (2018)

24. K. Sakagami, M. Morimoto, M. Yairi, 14th International Congress on Sound and Vibration, (Cairns, Australia, 2007) 